\title{
Cisticercose bovina em matadouro-frigorífico sob inspeção sanitária no município de Teixeira de Freitas-BA: prevalência da enfermidade e análise anatomopatológica de diagnósticos sugestivos de cisticercose
}

\section{Bovine cysticercosis in slaughterhouse under sanitary inspection in Teixeira de Freitas-Brazil: prevalence and anatomy pathological analysis of suspected cysticercosis diagnosis}

\author{
Davi de Oliveira Almeida, ${ }^{*}$ Horácio Peçanha Igreja, ${ }^{* *}$ Fernanda Martinez Xavier Alves, ${ }^{*}$ lacir Francisco dos Santos, ${ }^{* \star *}$ \\ Rogerio Tortelly***
}

\begin{abstract}
Resumo
Foram diagnosticados, nas linhas de inspeção e registrados nos mapas de condenação, 115 (4,20\%) casos de cisticercose bovina, 108 (92,17\%) considerados degenerados e 7 (6,08\%) viáveis, em 2778 bovinos oriundos de várias cidades da região sul da Bahia, Brasil, durante o mês de outubro de 2005. Oitenta (69,56\%) ocorreram no fígado, 19 (16,52\%) no coração e 16 $(13,91 \%)$ nos masseteres. Macroscopicamente, os cisticercos viáveis apresentavam um aspecto cístico e uma parede translúcida. Os considerados degenerados possuíam um aspecto caseoso a cáseo calcário, com diâmetro em torno de $0,5 \mathrm{~cm}$, coloração esbranquiçada a amarelada e consistência firme. Os 108 cisticercos considerados degenerados foram submetidos à análise microscópica, para a confirmação do diagnóstico macroscópico. A microscopia revelou que somente 17 amostras apresentaram lesões características indicativas de cistos degenerados, em 7 casos no fígado e 6 na musculatura, onde foram observadas estruturas ovóides e basofílicas, com as características dos corpúsculos calcários, enquanto em 4 casos foram observados fragmentos de cestóides. As lesões eram representadas por um processo inflamatório na maioria das vezes granulomatoso com o centro cáseo calcário. Concluiu-se que em casos de lesões inflamatórias e/ou mineralizadas torna-se muito difícil o inspetor diagnosticar tal enfermidade macroscopicamente.
\end{abstract}

Palavras-chave: bovino, cisticercose, corpúsculos calcários, histopatologia, inspeção sanitária.

\begin{abstract}
Were diagnosed, in inspection lines of SIF, and registered in the condemnation maps, $115(4.20 \%)$ cases of bovine cysticercosis, $108(92.17 \%)$ were considerate degenerate and 7 (6.08) viable, in 2778 bovines originating from many cities of south region of Bahia state-Brazil, during october 2005. In these cases, 80 (69.56\%) occurred in liver, $19(16.52 \%)$ in heart and $16(13.51 \%)$ in masseter muscles. Macroscopically the viable cysticerci had a cystic aspect and a translucent membrane. The cysticerci considerate degenerate had a caseous and/or caseous calcareous aspect, $0.5 \mathrm{~cm}$ of diameter, a white-yellowish color and a firm consistency. The 108 considered degenerate cysticerci were submitted a microscopic analysis, for the confirmation of the macroscopic diagnosis. The microscopic analysis revealed that only 17 samples showed lesions characteristics of the degenerate cysts, 7 cases in liver and 6 in musculature, where were observed ovoid and basophilic structures, with the characteristics of the calcareous corpuscles. In 4 cases were observed cestode fragments. The lesions were represented for an inflammatory process in many times granulomatoseus with the caseous calcareous center. Concluded that inflammatory / mineralized lesions make difficult the inspector macroscopically diagnosis of cysticercosis.
\end{abstract}

Keywords: bovine, cysticercosis, calcareous corpuscles, histopathology, sanitary inspection.

* Programa de Pós-Graduação em Medicina Veterinária (Doutorado) - Higiene Veterinária e Processamento Tecnológico de Produtos de Origem Animal da Faculdade de Veterinária da Universidade Federal Fluminense, Niterói, RJ, Brasil.

** Fiscal Agropecuário, Ministério da Agricultura, Teixeira de Freitas, BA, Brasil.

*** Departamento de Tecnologia dos Alimentos, Faculdade de Veterinária, Universidade Federal Fluminense. Rua Vital Brazil Filho, 64. Niterói, RJ. 24230-340. Brasil.

* Autores para correspondência: lacir Francisco dos Santos. E-mail: ifsantos@vm.uff.br e Davi de Oliveira Almeida. E-mail: davibber@yahoo.com.br. 


\section{Introdução}

O Serviço de Inspeção Federal (SIF), além da grande importância que representa como órgão fiscalizador em relação à saúde pública é, sem dúvida, aquele em que se pode projetar, através de seus relatórios, estudos epidemiológicos regionais e nacionais, sendo a principal fonte de dados da prevalência da cisticercose no país.

Uma importante medida a ser tomada, para o controle da cisticercose, é a identificação dos cistos ou da lesão pelos médicos veterinários visando a condenação dos tecidos então parasitados, interrompendo, assim, o ciclo do parasito e prevenindo a transmissão da enfermidade. Contudo, alguns aspectos tornam difíceis o seu diagnóstico e o trabalho executado por estes profissionais, já que o Cysticercus bovis pode ser encontrado em locais que não são considerados de eleição dos cestóides ou, principalmente, nos casos em que o parasito se encontra degenerado.

A cisticercose bovina é uma parasitose cosmopolita, cuja distribuição e prevalência são variáveis em diferentes áreas geográficas do mundo (Acha e Szyfres, 1986), e no Brasil, onde ela é enzoótica, assume, às vezes, caráter epizoótico (Santos, 1993b).

Santos (1993b) admite que a ocorrência da cisticercose no país é superior aos dados publicados. Uma pesquisa feita por Santos (1996) revelou que ocorrências regionais, no Brasil, registram percentuais de cisticercose bovina de $4 \%$ a $45 \%$ nas propriedades rurais, com alguns lotes chegando a $100 \%$. Ressalta ainda que prevalências acima de $10 \%$ são extremamente comuns e que a ocorrência na população é tão elevada quanto a mais elevada do mundo.

Em relação ao aspecto macroscópico, o cisticerco viável é cístico, com uma parede translúcida, onde se percebe o escólex no interior; já o cisticerco degenerado possui aspecto caseoso/caseo calcário, diâmetro em torno de $0,5 \mathrm{~cm}$, coloração esbranquiçada a amarelada e consistência firme (Santos 1984; Costa, 2003).

Na microscopia, o cisticerco viável é reconhecido por seu escólex com ventosas e corpúsculos calcários. As alterações causadas nestes casos, na maioria das vezes, se limitam a um infiltrado inflamatório discreto e ao deslocamento das células do tecido (Costa, 2003). Porém em casos de cisticercos degenerados, a lesão se apresenta granulomatosa, rica em histiócitos e células gigantes multinucleadas contínuas à área central, freqüentemente cáseo-calcária. A periferia da lesão pode exibir uma cápsula fibrosa, às vezes mineralizada (Santos et al., 2001; Tortelly, 2003). Nestes casos, é fundamental a observação dos corpúsculos calcários para a conclusão do diagnóstico. Estes corpúsculos são estruturas circulares ou ovóides, incolores e basofílicas, típicas dos cestóides (Chowdhury et al., 1962). Inclusive, podem fornecer a única evidência, no corte histológico, de que o espécime é um cestóide (Georgi e Georgi, 1988).

O diagnóstico e o destino dos tecidos realizado em matadouros, baseiam-se nas características macroscópicas dos cisticercos, de acordo com o artigo 176 do Regulamento da Inspeção Industrial e Sanitária dos Produtos de Origem Animal (Brasil,1997).

Contudo, como múltiplas lesões podem ter os mesmos aspectos macroscópicos, há possibilidade de ocorrerem dificuldades de diagnóstico, devendo-se, nesses casos, recorrerse a exames complementares, como o histopatológico.

\section{Material e métodos}

As amostras foram coletadas em um matadouro, sob inspeção federal, localizado no município de Teixeira de Freitas, sul do estado da Bahia, Brasil. As amostras foram coletadas em outubro de 2005 sendo abatidos, no período, 2.738 bovinos. Em comum acordo com o médico-veterinário - ocupante do cargo de fiscal sanitário do MAPA - foram coletadas 115 amostras de fígados e musculatura (esquelética e cardíaca) com lesões de cisticercose, diagnosticadas na linha de inspeção e registradas nas papeletas de condenação do SIF. Estas amostras foram devidamente identificadas em ficha própria, acondicionadas em formol a $10 \%$ e, enviadas para o serviço de Anatomia Patológica Prof. Jefferson Andrade dos Santos na Universidade Federal Fluminense onde foram processadas pela técnica habitual para inclusão em parafina e coloração pela hematoxilina-eosina (H.E.), para exame histológico.

Todas as amostras foram analisadas macroscopicamente, sendo que, aquelas em que o cisticerco se apresentou viável (íntegro), foram descartadas da análise microscópica por apresentarem características para um diagnóstico macroscópico definitivo. A análise microscópica foi somente realizada naquelas lesões consideradas cisticercose, mas de difícil diagnóstico macroscópico.

\section{Resultados}

Considerando o número de animais abatidos e o número de casos diagnosticados na linha de inspeção observou-se uma prevalência de 4,20\% para a enfermidade.

Foi registrada maior ocorrência de cisticercose no fígado, seguida do coração e do masseter, sendo 108 casos $(93,92 \%)$ degenerados (Quadro 1).

Quadro 1: Características da cisticercose bovina, observadas pelo serviço de inspeção sanitária, num matadouro em Teixeira de Freitas-BA, Brasil, 2005.

\begin{tabular}{|c|c|c|c|c|}
\hline Localização & Aspecto & $\begin{array}{c}\mathrm{N}^{0} \text { de animais } \\
\text { infectados }\end{array}$ & \multicolumn{2}{|c|}{$\%$} \\
\hline \multirow{2}{*}{ Fígado } & Viável & 2 & 1,74 & \multirow{2}{*}{69,56} \\
\hline & Degenerado & 78 & 67,82 & \\
\hline \multirow{2}{*}{ Coração } & Viável & 5 & 4,35 & \multirow{2}{*}{16,52} \\
\hline & Degenerado & 14 & 12,17 & \\
\hline \multirow{2}{*}{ Masséter } & Viável & 0 & - & \multirow{2}{*}{13,91} \\
\hline & Degenerado & 16 & 13,91 & \\
\hline Total & & 115 & & \\
\hline
\end{tabular}

Nas análises macroscópicas, observou-se que em sete casos estudados, o cisticerco estava viável (íntegro) e evidenciava um aspecto cístico, com uma parede translúcida e o escólex no interior. Em 108 casos o cisticerco estava degenerado e possuía aspecto caseoso / cáseo-calcário, diâmetro em torno de $0,5 \mathrm{~cm}$, coloração esbranquiçada a amarelada e consistência firme.

Dos 108 casos analisados microscopicamente, somente 17 apresentaram características da parasitose (Tabela 1). 
Tabela 1: Casos confirmados de $C$. bovis em material oriundo de um matadouro em Teixeira de Freitas-BA, 2005

\begin{tabular}{cccc}
\hline \hline Amostras & \multirow{2}{*}{$\begin{array}{c}\text { Casos estudados } \\
\text { microscopicamente }\end{array}$} & \multicolumn{2}{c}{ Casos confirmados } \\
\cline { 3 - 4 } & 14 & $\mathrm{~N}$ & $\%$ \\
\hline Coração & 16 & 6 & 21,42 \\
Masséter & 78 & 8 & 37,5 \\
Fígado & 108 & 17 & 10,25 \\
\hline Total & &
\end{tabular}

As amostras que não foram confirmadas na microscopia foram, na sua grande maioria, diagnosticadas como migração por outras larvas de helmintos (86 casos). Também houve cinco casos em que a histopatologia evidenciou um processo inflamatório inespecífico (tabela 2).

As lesões hepáticas apresentaram um processo inflamatório ora granulomatoso, ora abscedante. O primeiro era constituído por lesões nodulares ricas em histiócitos, células gigantes tipo Langhans e tipo corpo estranho, células epitelióides contínuas à área central, freqüentemente cáseo-calcária. A periferia apresentava uma cápsula fibrosa às vezes com mineralização de aspecto linear ou em placas (Figura 1), invadida por um infiltrado linfo eosinofílico. A lesão abscedante era rica em restos celulares envoltos por uma cápsula fibrosa onde era observado um infiltrado de mononucleares e eosinófilos, com predomínio dos últimos.
Tabela 2: Diagnósticos, após a histopatologia, em material suspeito de cisticercose bovina, registrados pelo serviço de inspeção federal em Teixeira de Freitas-BA, 2005.

\begin{tabular}{ccc}
\hline \hline Diagnósticos histopatológicos & $\mathrm{N}^{\circ}$ de casos & $\%$ \\
\hline Cisticercose & 17 & 15,74 \\
Migração de larvas de helmintos & 86 & 79,62 \\
Processo inflamatório inespecífico & 5 & 4,62 \\
\hline Total & 108 & 100 \\
\hline \hline
\end{tabular}

As lesões encontradas na musculatura possuíam um centro ora cáseo-calcário, ora rico em detritos celulares. Contínua a esta área, notou-se freqüentemente histiócitos em paliçada, células multinucleadas e células epitelióides. A periferia da lesão encontrava-se fibrosada e invadida por mononucleares e eosinófilos (Figura 2).

Em 7 casos no fígado e em 6 casos na musculatura (5 no masséter e 1 no coração) foram encontradas estruturas ovóides e circulares levemente basofílicas, com as características dos corpúsculos calcários (Figura 3). Em 4 casos (1 no fígado, 2 no coração e 1 no masséter) foram observados fragmentos de cestóides (Figura 4). Em 1 caso foi notada a presença de segmentos de larvas de nematóide em meio a restos celulares no fígado.

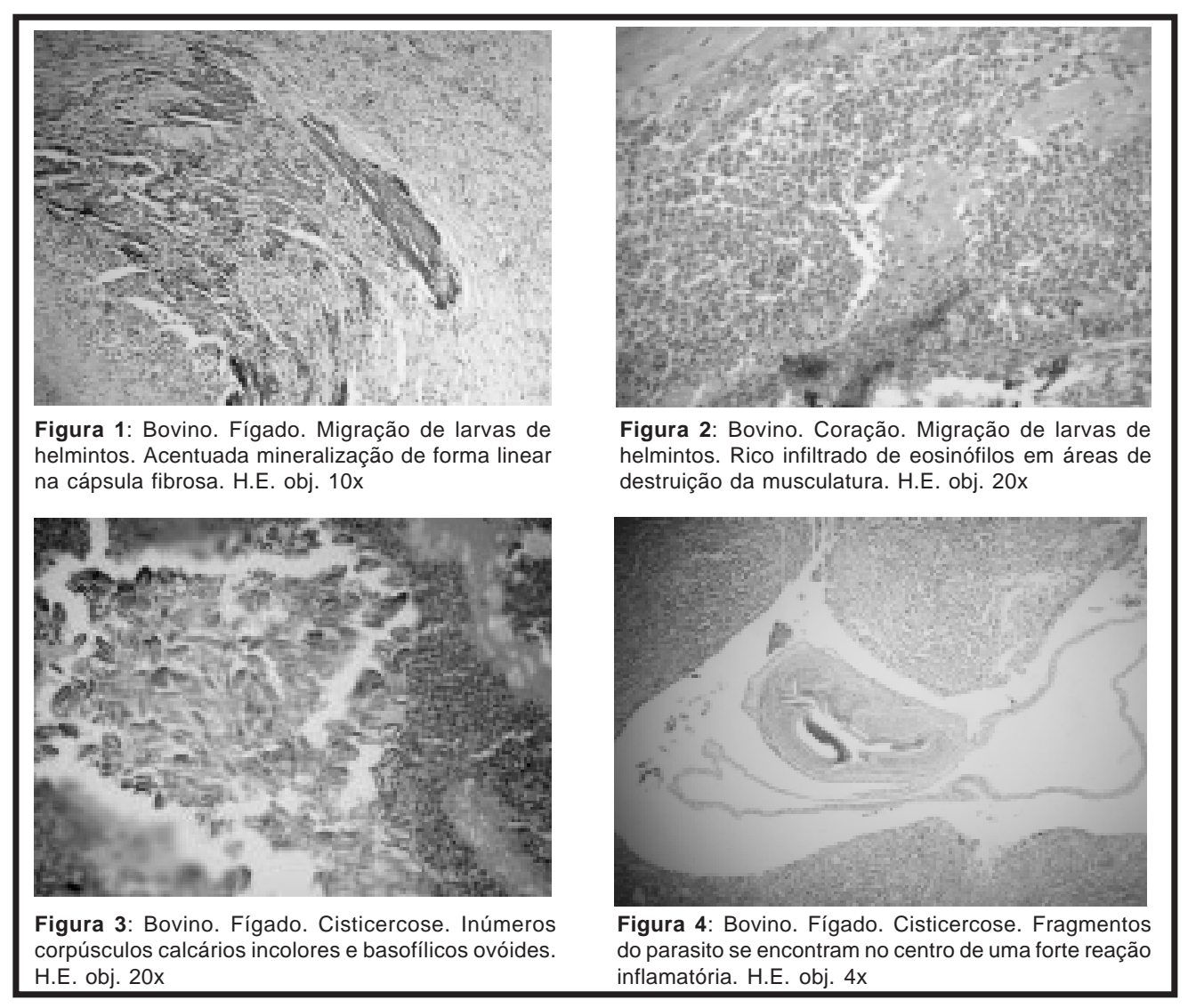




\section{Discussão}

Objetivando avaliar a prevalência da cisticercose no Brasil baseado em dados fornecidos pelo SIF, vários autores registram diversos percentuais. Os mais recentes são $5,81 \%$, no estado do RJ (Santos, 2002); em MG, 3,20\% (Reis e Raghiante, 2000) e 6,90\% (Almeida et al. 2002); em SP, 2,97\% (Fernandes et al. 2002); 5,10\% em animais oriundos de SP, MG e GO (Santos, 1993a) e 4,12\% no Brasil central (Santos, 1993b). O presente estudo mostrou uma prevalência de $4,20 \%$, o que demonstra certa conformidade com os dados citados pela literatura.

No que diz respeito ao aspecto da lesão pelo $C$. bovis, há uma maior ocorrência do cisticerco degenerado, fato descrito anteriormente por diversos autores, como Santos et al. (2001) 83,50\%; Almeida et al. (2002) 64,12\%, Costa (2003) 88,46\%. Este fato foi também observado neste estudo, onde se encontrou uma prevalência de $93,92 \%$ para o cisticerco degenerado, de acordo com registros do SIF. Porém estes achados estão em desacordo com os de Minozzo et al. (2002), que relataram uma maior ocorrência de cisticercos viáveis após a inspeção $(81,20 \%)$, tratando-se, entretanto, de um trabalho experimental cuja contagem foi feita 90 dias após a inoculação.

Em relação à localização do cisticerco nos animais o coração, seguido dos músculos mastigatórios, são os locais mais comumente afetados (Santos, 1993a; Manhoso, 1996). No entanto, como terceiro sítio de localização, Santos (1984) relata o diafragma, Santos (1993a) os pilares do diafragma e Manhoso (1996) o esôfago. Contudo Almeida et al. (2002) e Santos (2002) relatam que o cisticerco é mais freqüentemente localizado nos músculos mastigatórios, seguido do coração. Digno de registro foram as observações de Minozzo et al. (2002) que citam uma maior freqüência dos cisticercos na musculatura dianteira $(46,0 \%)$, musculatura traseira $(35,33 \%)$, coração $(6,98 \%)$ e músculos da mastigação $(3,56 \%)$. Porém, o registro das linhas de inspeção do SIF, neste estudo assinala que as lesões tiveram a seguinte distribuição: fígado $(69,56 \%)$, coração $(16,52 \%)$ e masséter $(13,91 \%)$.

Apesar do fígado não ser um dos locais de predileção do cisticerco, alguns autores (Minozzo et al., 2002) relatam uma alta prevalência neste órgão, enquanto outros fazem menção ao fato (Tortelly, 2003).

Poucos autores descrevem o aspecto macroscópico do cisticerco degenerado. Santos (1984) o descreve como sendo caseoso algumas vezes, com massa amarelada ou esverdeada. Costa et al. (2002), Costa (2003) e Tortelly (2003) o descrevem como sendo circulares, de consistência firme, diâmetro em torno de $0,5 \mathrm{~cm}$, coloração amarelada e com aspecto ora caseoso, ora calcário. Tais observações estão de acordo com o material examinado neste estudo.

Quanto à microscopia, há concordância que a lesão seja representada por um granuloma (Reis, 1980; Santos et al., 2001; Tortelly, 2003) que por vezes se apresenta com centro necrótico e/ou mineralizado (Reis, 1980; Costa, 2003; Tortelly, 2003). Circundando a área central, há presença de histiócitos dispostos em paliçada (Reis, 1980; Santos et al., 2001; Tortelly, 2003), de células gigantes multinucleadas (Reis, 1980; Santos et al., 2001; Tortelly, 2003), ora tipo Langhans, ora tipo corpo estranho (Tortelly, 2003). Segundo Santos et al. (2001) o infiltrado inflamatório é de polimorfonucleares, porém Tortelly
(2003) afirma que o infiltrado é misto, enquanto Costa (2003) relata que o infiltrado é de mononucleares. Contudo, estes autores concordam que há uma forte infiltração de eosinófilos no processo inflamatório. Um fato interessante é a calcificação da cápsula fibrosa citada por alguns autores. Costa (2003) relata que a cápsula fibrosa possui características de tecido de granulação e mineralização, já Tortelly (2003) evidenciou o aspecto linear mineralizado, tanto na cápsula, como nas imediações da lesão. Tais fatos foram também observados em nossos estudos.

Fato importante é que os autores citados realizaram uma análise microscópica de diagnósticos presuntivos diferentes, porém seus achados foram semelhantes. Gibson (1959), Santos et al. (2001) e Costa (2003) estudaram lesões suspeitas de cisticercose, Reis (1980) estudou alterações hepáticas que, microscopicamente, foram diagnosticadas como migração de larvas de helmintos e Tortelly (2003) estudou lesões hepáticas em que o diagnóstico presuntivo foi a hidatidose. Isto mostra a semelhança entre as lesões de aspecto caseoso/cáseo-calcário, tanto no aspecto macro, quanto no microscópico. Assim, os dados apresentados pelos Serviços de Inspeção Sanitária podem apresentar distorções, em virtude da falta de um apoio laboratorial complementar, o que dificulta a determinação de valores reais para determinada enfermidade, como ocorreu neste estudo. Uma característica importante a ser observada é a presença de estruturas denominadas corpúsculos calcários, pois na ausência destes, as lesões caseosas e/ou mineralizadas suspeitas de cisticercose ou migração de outras larvas de helmintos são idênticas. Nestes casos o diagnóstico histopatológico é de grande valia para a diferenciação destas lesões, e até mesmo para com outras lesões, como a da tuberculose, conforme descreve Kelly (1997).

Segundo Chowdhury et al. (1962), Pawlowski et al. (1988) e Georgi e Georgi (1988) os corpúsculos calcários são estruturas típicas dos cestóides, e de acordo com Georgi e Georgi (1988) estes podem fornecer a única evidência, no corte histológico, de que o espécime é um cestóide. Estudos realizados por Santos (1984), Santos et al. (2001), Costa (2003) confirmaram a presença dos corpúsculos calcários em musculatura bovina, enquanto Costa et al. (2002) e Tortelly (2003) os observaram no fígado.

Santos et al. (2001) e Costa (2003) caracterizam os corpúsculos como sendo ovóides, enquanto Tortelly (2003) esféricos ou ovóides. Quanto à coloração, Santos et al. (2001) citam que são ora claros, ora acidófilos, Costa (2003) cita que são elementos basofílicos, acidófilos e incolores e Tortelly (2003) incolores e basofílicos, tendo os circulares características psamomatosas. Chowdhury et al. (1962) e Pawlowski et al. (1988) comentam que alguns corpúsculos têm características de anéis concêntricos. Em relação à composição dos corpúsculos, Chowdhury et al. (1962) descrevem a presença de cálcio, glicogênio, proteínas e fosfatase alcalina, Pawlowski (1988) cálcio, fósforo, silício e zinco. Esta constituição parece indicar a variação da afinidade tinturial dos corpúsculos calcários.

A dificuldade de diagnóstico definitivo da parasitose em lesões inflamatórias e mineralizadas, macroscopicamente, pode ocorrer em razão da multiplicidade de alterações similares ocasionadas por vários agentes etiológicos, levando a altos índices de registros de diversas enfermidades nas papeletas de condenação. 


\section{Conclusão}

De acordo com os resultados, pode-se concluir que nos casos de lesões inflamatórias e/ou mineralizadas é difícil o diagnóstico macroscópico da enfermidade, sendo que nos

\section{Referências}

ACHA, P. N.; SZYFRES, B. Zoonosis y enfermedades transmisibles comunes al hombre y a los animales. 2. ed. Washington: OPAS, 1986, $989 \mathrm{p}$.

ALMEIDA, L. P.; MOREIRA, M. D.; REIS, D. O.; SANTOS, W. L. M. Cisticercose bovina: Um estudo comparativo entre animais abatidos em frigoríficos com serviço de Inspeção Federal e com Inspeção Municipal. Higiene Alimentar, São Paulo: L.F.G.S. Higiene Alimentar Publicações e Serviços LTDA, v. 16, n. 99, p. 51-55, ago. 2002.

BRASIL. Ministério da Agricultura. Lei 1.283 de 18/12/1950, regulamentada pelo decreto 30.691 de 20/03/1952 e alterado pelo Decreto 1.255, de 25/06/1962. Regulamento de Inspeção Industrial e Sanitária de Produtos de Origem Animal. RIISPOA, 1997.

CHOWDHURY, A. B.; DASGUPTA, B.; RAY, H. N. On the nature and structure of the calcareous corpuscles in Taenia saginata. Parasitology, Cambridge: Cambridge University Press, v. 52, p. 152157, 1962.

COSTA, R. F. R. Pesquisa de cisticercose e caracterização das reações inflamatórias em corações de bovinos comercializados na cidade de Nova Friburgo/RJ, inspecionados pelas técnicas de Santos (1976) e do fatiamento. 2003. 63 f. Dissertação (Mestrado em Higiene Veterinária e Processamento Tecnológico de Produtos de Origem Animal) - Faculdade de Veterinária, Universidade Federal Fluminense, Niterói, 2003.

COSTA, R. F. R.; SANTOS, I. F.; TORTELLY, R. Cisticercose hepática bovina. In: XXIX Congresso Brasileiro de medicina veterinária, 2002, Gramado. Anais... Gramado: Sovergs, 2002.

FERNANDES, J. O. M.; SILVA, C. L. S. P.;BORGES, J. H. R.; PEGAIANE, J. C.; COELHO, R. V. Prevalência da cisticercose bovina em animais abatidos em estabelecimento sob regime de Inspeção Federal no município de Andradina-SP. Ciência Agrária e Saúde, Andradina: FEA, v. 2, n. 1, p. 14-17, jan./jun., 2002.

GEORGI, M. E.; GEORGI, J. R. Diagnóstico histopatológico. In: GEORGI, J. R. Parasitologia Veterinária. 4. ed. São Paulo: Manole Ltda, 1988. 370 p. cap. 14. p. 335-365.

GIBSON, T. E. The identification of Cysticercus bovis, with special reference to degenerate cysticerci. Annals of Tropical Medicine Parasitology, London: Maney Publishing, v. 53, p. 25-26, 1959.

KELLY, W. R. The Liver and Biliary Sistem: helmintic infections of liver and bile ducts. In: JUBB, K. V. F.; KENNEDY, P. C. and PALMER, N. Pathology of Domestic Animals. 4. ed. San Diego, Cal.: Academic Press, 1997. 3 v., v. 2, cap. 2, 747 p.

MANHOSO, F. F. R. Prevalência de cisticercose bovina em animais abatidos no município de Tupã, SP (1992-1993). Higiene Alimentar, São Paulo: L.F.G.S. Higiene Alimentar Publicações e Serviços LTDA, v. 10, n. 45, p. 44-47, set./out., 1996. estudos sobre a cisticercose deve-se incluir, também, a pesquisa dos corpúsculos calcários. E, finalmente, observouse que os dados avaliados no país sobre a cisticercose podem estar superestimados, merecendo uma atenção maior pelos órgãos de fiscalização.

MINOZZO, J. C.; GUSSO, R. L. F.; CASTRO, E. A.; LAGO, O.; SOCCO, V. T. Experimental bovine infection with Taenia saginata eggs: recovery rates and cysticerci location. Brazilian Archives of Biology and Technology, Curitiba: Tecpar, v. 45, n. 4, dez., 2002.

PAWLOWSKI, I. D.; YAP, K. W.; THOMPSON, R. C. A. Observation and structure of calcareous corpuscles in Taeniid cestodes. Parasitology Research, Berlin: Springer-Verlag Heidelberg, v. 74, p. 293-296, 1988. REIS, A. C. F. Estudo histopatológico das alterações hepáticas observadas em bovinos azebuados abatidos nos estados de Goiás e Paraná-Brasil. Rio de Janeiro, 1980, 100p. Dissertação (Mestrado em Ciências - Área de Patologia Animal) - Decanato de pesquisa e pós-graduação, Universidade Federal Rural do Rio de Janeiro, Seropédica-RJ. 1980.

REIS, D. O.; RAGHIANTE, F. Cisticercose bovina: tendência da doença em animais abatidos em um frigorífico de Uberlândia, MG, sob inspeção federal, 1994-1998. Higiene Alimentar, São Paulo: L.F.G.S. Higiene Alimentar Publicações e Serviços LTDA, v. 14, n. 70, p. 20-22, março, 2000.

SANTOS, I. F. Diagnóstico da cisticercose bovina em matadouros. III Exame dos pilares diafragmáticos. Higiene Alimentar, São Paulo: L.F.G.S. Higiene Alimentar Publicações e Serviços LTDA, v. 7, n. 25, p. 26-34, março, 1993a.

SANTOS, I. F. Diagnóstico da cisticercose bovina em matadouros: novas técnicas de exame de esôfago e diafragma. 1984. 127 f. Tese (Doutorado em Medicina Veterinária) Faculdade de Saúde Pública, Universidade de São Paulo, São Paulo. 1984.

SANTOS, I. F. O Cysticercus bovis (forma larvar da Taenia saginata) pode infectar o homem? Higiene Alimentar, São Paulo: L.F.G.S. Higiene Alimentar Publicações e Serviços LTDA, v. 10, n. 44, p. 13-14, 1996.

SANTOS, I. F. Um modelo de inspeção para a detecção da cisticercose muscular bovina em matadouros. 1993. 73 f. Tese (Concurso para professor titular) - Departamento de Tecnologia de Alimentos, Universidade Federal Fluminense, Niterói, 1993b.

SANTOS, I. F.; MANO, S. B.; TORTELLY, R.; SANTOS, M. L. S.; SILVA, D. A. S. Estudo da localização do Cysticercus bovis em corações de bovinos abatidos sob inspeção. Higiene Alimentar, São Paulo: L.F.G.S. Higiene Alimentar Publicações e Serviços LTDA, v. 15, n. 89, p. 37-44, 2001.

SANTOS, R. E. V. Exame post mortem pela técnica de Santos, no estudo da prevalência da cisticercose bovina em matadouros do estado do Rio de Janeiro, comparada à utilizada pelo serviço de inspeção estadual. 2002. 64 f. Dissertação (Mestrado em Medicina Veterinária) - Faculdade de Veterinária, Universidade Federal Fluminense, Niterói. 2002.

TORTELLY, R. Lesões em fígados de bovinos sob Inspeção Sanitária e sua importância em Saúde Pública. 2003. 94 f. Tese (Doutorado em Ciências Veterinárias) - Instituto de Veterinária, Universidade Federal Rural do Rio de Janeiro, Seropédica. 2003. 